\title{
THE DEVELOPMENT OF FRACTURE MECHANICS HYPOTHESES APPLICABLE TO THE CALCULATION OF REINFORCED CONCRETE STRUCTURES FOR THE SECOND GROUP OF LIMIT STATES
}

\author{
Igor Anatolyevich lakovenko* \\ National Aviation University, Kiev, Ukraine \\ Vladimir Ivanovich Kolchunov \\ South-West State University, Kursk, Russia
}

The problems of the fracture mechanics hypotheses development are formulated with reference to the calculation of reinforced concrete structures in the presence of cracks. The main aspects of fracture mechanics are given. They focus on the features of the predestruction zone, as well as the hypotheses and assumptions underlying the calculation of reinforced concrete structures for the second group of limit states. It is described the features of cutting a two-cantilever element including a crack for constructing an effective instrument of calculation for reinforced concrete with allowance for physical nonlinearity, cracking processes, bond of reinforcement with concrete, and the effect of discontinuity. It is obtained a new solution to the problem of the stressed-strained state of the reinforced concrete element in the zone immediately adjacent to the crack.

Key words: Hypotheses, Fracture mechanics, Reinforced concrete structures, New concrete constants, Double-cantilever element, Calculation apparatus, Crack formation, Bond, Effect of discontinuity

\section{INTRODUCTION}

The main characteristic feature of reinforced concrete is the presence of cracks in resisting its force and deformation effects. Reinforced concrete structures, as a rule, are used in the stage after the cracks emergence (only the width of their opening is limited). Therefore, it is natural to strive to study the stress-strain state in the vicinity of the crack. So, it is a problem that is investigated by the fracture mechanics, actively developing in recent years [01-04].

The results achieved in this area allow already today to extend the accumulated information to the calculation of reinforced concrete [01, 02, 05]. It should be borne in mind that for concrete as an elastoplastic material, laws of linear fracture mechanics are not applicable.

The specificity of the material manifests itself not only in the basic dependence of the fracture mechanics, which relates the stress intensity factor to the amount of released energy per unit of the newly created crack surface zl, but also in such concepts as the prefracture zone at the crack mouth, the critical stress intensity factor $\mathrm{Kbr}$ and the corresponding value $\mathrm{zbr}$, the limiting value of zbu, corresponding to continuation of the crack, etc. Much depends on the successful separation of the two-cantilever element, including the crack [03, 05-08].

The main aspects of fracture mechanics emphasizing the features of predestruction zone of and communications between key parameters of $\mathrm{KI}$, I with the localized deformation of in this zone. It follows from their analysis $[01,03,05$, etc.] that:

1 ) the two surfaces of the crack should close smoothly, in order to avoid an unreasonable increase in stresses in the end zone to infinity;

2) due to the large curvatures of the closing surfaces in the prefracture zone, surface tension forces that newly crimping the formed crack surface to each other;

3 ) according to the Barenblatt's postulate [9], the measure of the interaction forces in the 
1) predestruction zone is a constant for a given material when it is elastic. Accounting the elastoplastic resistance requires the introduction of additional constants.

Below is shown, based on the noted aspects and their analysis, the development of hypotheses of fracture mechanics in relation to reinforced concrete structures was carried out.

\section{PREDESTRUCTION ZONE HYPOTHESES AND PREMISES}

A fracture in the form of an elongated ellipse is modeled, at the tip of which there is a predestruction zone. The predestruction zone is a continuation of a fracture in the area where forces distributed along the shores of the crack are distributed according to a certain law.

If we take the parabolic law for describing the shores of the crack in this section (Figure 1), then, having the diagram $s-\omega$, we can obtain the distribution of the interaction force along the length of the end zone. The parabolic surfaces of the continuum, inverted convex inward, can be idealized (for the model medium) as surfaces with a large radius of curvature, in which a surface tension arises that causes compression along the interface line of surfaces (Figure 1). The resulting forces, summing up with the stresses arising from tensile forces, form the total forces acting along the shores of the predestruction zone.

Moreover, considering that the hyperbolic surfaces of the continuum smoothly close at point A (Figure 1), all the stresses at this point are excluded. The considered scheme of the predestruction zone allows, although approximately, to explain the origin of the forces of interaction and their different directions in the end zone. Determination of the exact shape of the surface of the zone is a very complex task of the elastoplastic continuum (today there are only a few results of this solution for the simplest cases). By the way, the calculated Dugdale's curve, obtained from the solution by the Muskhelishvili method, qualitatively coincides with the one shown in Figure 1.

An exact solution of this problem will have little effect on the practical results of the calculation, since the dimensions of the predestruction zone for concrete are small (as will be shown below). Moreover, the maximum stresses in this zone do not exceed the value of Rbt, therefore the contribution of the stress state of the zone to the general stress state of the cross-section of the reinforced concrete element is not very noticeable. Nevertheless, the mechanism of crack initiation (with its development) is laid precisely in this zone, and therefore its detailed consideration is of paramount importance.

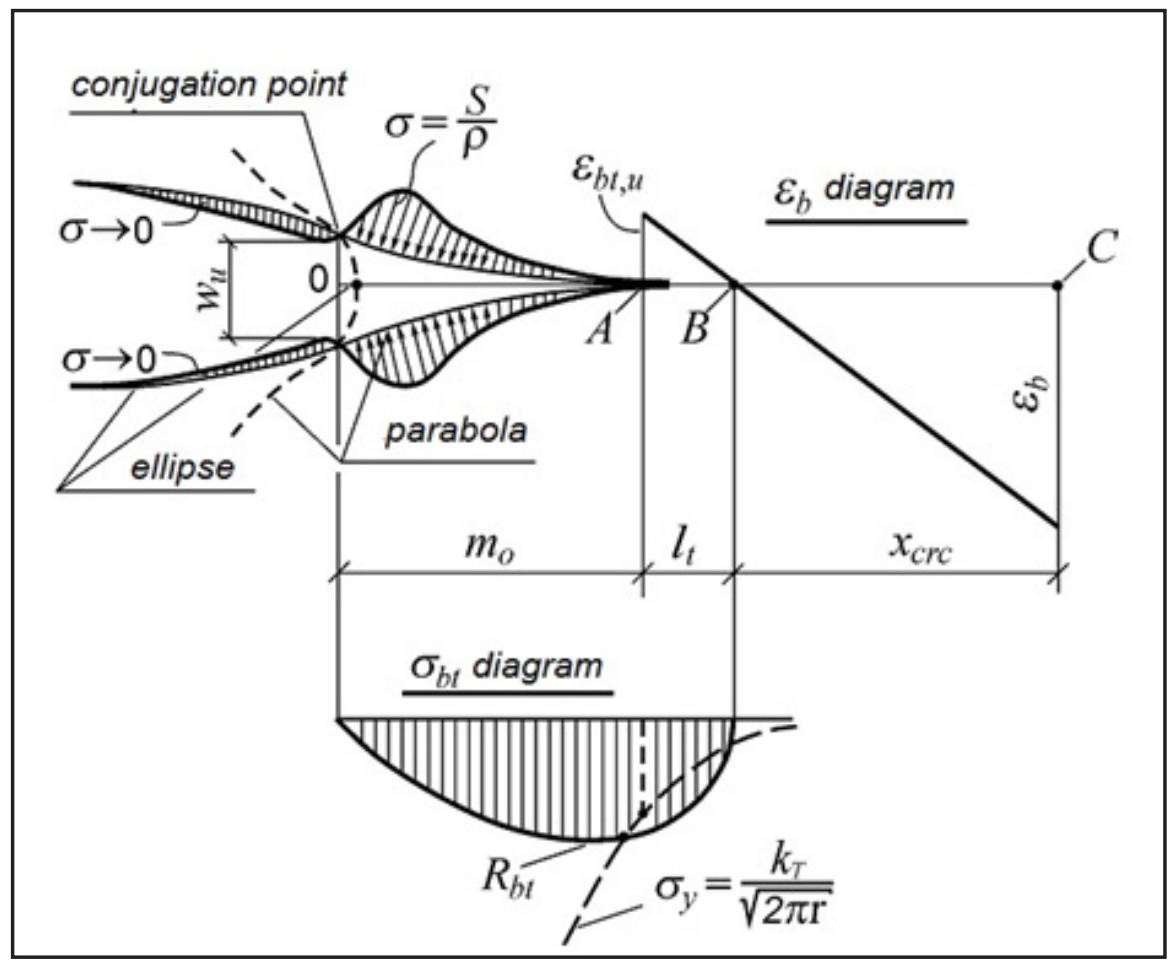

Figure 1: To analyze of the predestruction zone 
The number of studies have established that the parameter characterizing the crack initiation is the magnitude of the critical crack opening, designated by different authors in different ways: Wmax - in the Sih's model (Evanston, USA) [10], wmax - in the Hillerborg-Moder-Peterson's model - (Lund, Sweden) [11], b - in the LeonovPanasyuk's model, etc. The determination of this quantity by the majority of authors is carried out identically - on the basis of tests for axial extension (Sih's experiments [10], Hillerborg [11], Bažant $[01,12]$, etc.). The peculiarity of these tests is that the test unit allows you to set the amount of displacements, and, consequently, to obtain complete diagrams $s-\omega$ with a descending branch of deformation. And phenomenologically, it is believed that such diagrams can be used in the predestruction zone. Indeed, even an approximate analysis of these zones shows that along with tensile stresses there are also compressive forces that are successfully modeled in the noted tests for axial tension.

Thus, the processes can be considered identical occurring in the prism and in the corresponding fiber of the predestruction zone.

It can be formulated the following main hypotheses and assumptions, which are the basis for calculating reinforced concrete elements, based on the above analysis:

1. The profile of the crack mouth is modeled by an ellipse, matched in the prefracture zone with two parabolic surfaces smoothly closing at the end of the zone, so that there is no any stresses feature here.

2. The material in the prefracture zone is in a uniaxial stress state and possesses certain properties described by the sb- $\omega$ b diagram obtained in experiments on simple stretching (a hypothesis similar to that adopted in such models as Hillerborg-Moder-Peterson's [11], Sih's [10], Bažant $[1,12]$, Leonov-Panasyuk's, etc.), and the diagram Kb2-zb, which follows from the diagrams $\mathrm{sb}-\mathrm{eb}, \mathrm{sb}-\omega \mathrm{b}$ (as analogous dependencies for nonlinear deformation of concrete and after disturbance of its continuity).

3. The crack initiation occurs after reaching the width of crack's opening at the start of the predestruction zone of a certain critical value $\omega \mathrm{u}$ (considered as the material constant) equal to the limiting displacement in the $s-\omega$ diagram (a hypothesis similar to that used in the Sih's [10], Bažant [12], Hillerborg-ModerPeterson's models [11], etc.).
From the first and second hypotheses it follows that the stress diagram in the prefracture zone is described by a square parabola with a vertex at point $A$ (Figure 1). The length of the predestruction zone $m_{0}$ is determined from the condition that there are no stress characteristics at the point A:

$\sigma_{y}=R_{b t}=\frac{k_{b R}}{\sqrt{2 \pi m_{0}}}$.

From (1) equitation, it follows that:

$$
m_{0}=\frac{k_{b R}}{\sqrt{R_{b t}^{2} 2 \pi}} .
$$

Now, it can be proceed to the description of the stress-strain state of the reinforced concrete element in the adjacent to the crack zone, if it is taken one more additional presupposition: for the stretched and compressed concrete deformations in the section with a crack, the hypothesis of flat sections is valid only for the area located beyond the predestruction zone.

The stress diagram of stretched concrete in a section with a crack at the section from the end of the predestruction zone to the neutral axis also has the form of a square parabola with a vertex at point $A$ (Figure 2) on the basis of an additional presupposition in combination with the adopted sbt-ebt diagram adopted in [03].

\section{BASIC PROVISIONS AND FEATURES OF TWO-CANTILEVER ELEMENT EXCISION, INCLUDING A CRACK FOR CONSTRUCTING THE CALCULATING DEVICE OF REINFORCED CONCRETE}

The stress-strain state is analyzed by the methods of the theory of elasticity and plasticity for a solid body, an elementary cube is identified. It describes the relationship between stresses and deformations at a point. Then, , the established link is integrated over the entire section on the transition to the cross-section. As a result, the problem reduces to differential equations, the exact solution of which, as a rule, is very difficult. The hypothesis of plane deformations is adopted for the whole cross section, which considerably simplifies the solution of the problem in the resistance of materials.

The methods developed in the theory of elasticity and plasticity, and resistance of materials are 
not applicable for a discontinuous body with a crack (where the continuity of the body is broken), when establishing the connection between stresses and displacements. Nevertheless, the use of the fundamental cross-section method for a material with cracks brings its positive results. This also applies to the approximate acceptance of the determination of the stress intensity factor, and it can also be used to allotment of special two-cantilever element that has found application in fracture mechanics.

The separation of such a two-cantilever element, including a crack, with its core reinforced concrete element has its own specificity [06, 07]. Firstly, it is allocated to the entire height of the crack, and not for some of its elementary section. Secondly, the forces in the sections passing at a distance $t$ from the crack must be related to the required parameters of the stress-strain state of the reinforced concrete element. Thirdly, we should not forget about the virtual movements of the dedicated consoles when the neutral axis of the reinforced concrete element is rotated, that is, cantilever support is not absolutely rigid. Thus, the separation of a two-cantilever element for reinforced concrete is a very important and difficult task.

It must be linked not only with the task of determining the stress-strain state of the cross-section of the reinforced concrete element, but also with the task of distributing the bond between the reinforcement and concrete, since the appearance of a crack in the solid body can be considered as a deformation effect that affects the bonding characteristics of reinforcement and concrete in the zones adjacent to the crack. With the aid of a two-cantilever element, the connection of its stressed-deformed state with the value of bu in the predestruction zone seems to be the most successful. In this case, the compliance of the crack's shores is determined by using conventional methods of structural mechanics, through which the value of bu can be expressed. Thus, a two-cantilever element is used as a link between the dependencies of the mechanics of a solid deformed body and the fracture mechanics.

The considerations presented above [03, 05-08] were used to distinguish the two-cantilever element shown in Figure 2. Here, the parameter $t$ (characterizing the size of the compressed concrete zone in the vicinity of the crack) in accordance with the Saint-Venant's principle and with investigations of the near reinforcement zone, involving semi-analytic and numerical methods, is to a first approximation equal to one and a half of the reinforcement diameter.

In the future, the value of $t$ is refined from the solution of the bond problem. The tensile stresses in the separating sections are distributed according to the law of a square parabola from the neutral axis to the point, where the sign of these stresses changes. At the same time, their maximum value is limited by the value of Rbt; therefore, in a significant area, the actual distribution of tensile stresses is close to the rectangle, regardless of the law of their distribution at the elastic stage. The compressive stresses are distributed along a triangle in these same cross-sections on the areas adjacent to the reinforcement.

The unknown parameter lc (Figure 2) is determined from the additional premise of deformation of the concrete area located beyond the predestruction zone:

$l_{t}=\frac{2 R_{b t} x_{c r c}}{E_{b} \varepsilon_{b}}$,

where eb - strain of fiber compression concrete in the reinforced concrete element in a crosssection with a crack.

In turn, the parameter xcrc is determined from geometric considerations:

$x_{c r c}=h_{0}-h_{c r c}-l_{t}$.

Substituting (3) into equation (4) and performing algebraic transformations, we obtain:

$$
x_{c r c}=\frac{h_{0}-h_{c r c}-m}{1+\frac{2 R_{b t}}{E_{b} \varepsilon_{b}}}
$$

From the equality moments of all forces to zero relative to the point $\mathrm{O}$ (Figure 2, b), we obtain:

$$
\begin{gathered}
M_{c o n}=-\Delta T h_{c r c}+0,5 \sigma_{b t}^{\prime} b t\left(h_{c r c}-\frac{1}{3} t\right)- \\
-0,5 R_{b t} b\left(h_{c r c}-t-m\right)^{2}-\frac{2}{3} R_{b t} b m\left(h_{c r c}-t-\frac{2}{3} m\right) .
\end{gathered}
$$

The crack length hcrc is determined from the known stress intensity factor kbr, the equation for which is written using the cross-section method:

$$
h_{c r c}=\frac{1}{3} m+\frac{2}{3} m+t-\frac{\Delta T}{R_{b t} b}+\frac{0,5 \sigma_{b t}^{\prime} t}{R_{b t}} \text {. }
$$


Note, that in the first approximation (or as the first step in the iterative process), it must be taken

$$
h_{c r c} \approx h_{c r c, 1}=h_{c}-m-x_{c r c, 1} \text {, }
$$

\section{double-cantilever element}
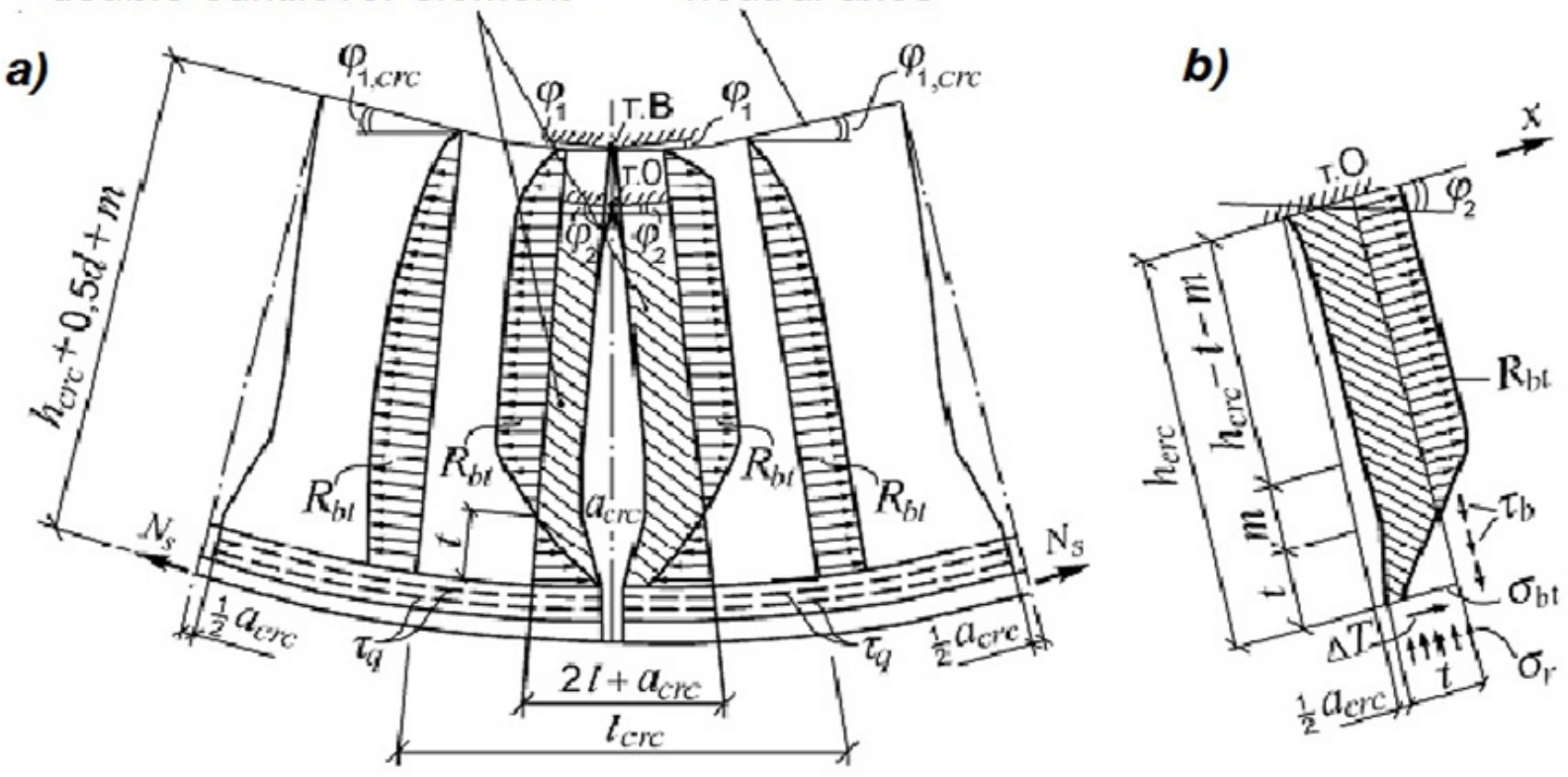

Figure 2: To realization of fracture mechanics dependencies in reinforced concrete:

a - characteristic diagrams of stresses in stretched concrete and excision of a special double-cantilever element in the vicinity of the crack; $b$ - the compliance calculating of the console
The value of the parameter $t$ is refined according to the condition that the moment M3 (Figure 3) is equal to the moment Mcon.

This condition follows from the attenuation of the tangential stresses $\mathrm{Tb}(\mathrm{z})$ (see Figure 2, b) as far as distance from the near reinforcement zone. In the area of constant moments, the transverse force is zero in the console, and therefore the tangential stresses $\mathrm{Tb}(\mathrm{z})$ are zero too.

Then,

$$
t=f\left(\sigma_{b t}^{\prime}, h_{c r c}, m, R_{b t}, b, \Delta T\right) \text {. }
$$

To determine the unknown $\Delta T$, we use the expression $\varphi b u$ as a function of compliance. This function is found from the definition of the energy rate of release:

$$
\varphi_{b u}=\lim _{\delta A \rightarrow 0}\left(\frac{\delta W-\delta V}{\delta A}\right)=\frac{d W}{d A}-\frac{d V}{d A},
$$

where bV - is the reduction of the potential energy of the body when the crack moves to a small increment bA; bW - is an extra work, which have done on the body while the crack is moving to a small increment bA; $A$ - is an area formed of crack. wher $\mathrm{xm}$ - the average height of the compressed zone of concrete in the area between the cracks in the rod reinforced concrete element.
Analysis of the "force - displacement" dependences for influences on the isolated two-console element. It shows that such dependencies are nonlinear and may even have a descending branch of deformation.

The area of diagrams, through which the value of the potential energy is expressed, differs from $0.5 \mathrm{P} 0 \mathrm{e} 0$. Here PO is a generalized force, and $\mathrm{e} 0$ is a generalized displacement. The integrals characterizing the areas of these diagrams give fairly close values to the value of $(2 / 3) \mathrm{P} 0 \mathrm{e} 0$, therefore the expression for the potential energy stored in the body can be represented as:

$$
V=\frac{2}{3} P_{0} e_{0} \text {. }
$$

The compliance of the $\mathrm{C}$ element is determined by the relation:

$$
e_{0}=C P_{0} \text {. }
$$

Then,

$\frac{d V}{d A}=\frac{4}{3} C P \frac{\partial P}{\partial A}+\frac{2}{3} P^{2} \frac{\partial C}{\partial A}$. 
Similarly, we transform the term . Then, substituting expression (13) into equitation (10), we have:

$$
\zeta_{b u}=\frac{1}{3}\left(P^{2} \frac{\partial C}{\partial A}-C P \frac{\partial P}{\partial A}\right) .
$$

With reference to the selected double-cantilever element, which is under the influence of five forces ( $\Delta T, P 1, P 2, q$, Mcon), the expression (14) takes next form:

$$
\begin{array}{r}
\Delta_{I}=\delta_{I}+\frac{1}{E_{b} I_{\text {con }}}\left[\frac{M_{4}+M_{5}}{2}\left(h_{c r c}-t-\frac{5}{8} m\right)\left(-M_{\text {con }}\right)\right] ; \\
\Delta_{I I}=\delta_{I I}+\frac{1}{E_{b} I_{\text {con }}}\left[\frac{M_{6}+M_{7}}{2}\left(h_{c r c}-t-\frac{5}{8} m\right)\left(-M_{\text {con }}\right)\right] ; \\
\Delta_{I I I}=\delta_{I I I}+\frac{1}{E_{b} I_{\text {con }}}\left[\frac{M_{8}}{2}\left(h_{c r c}-t-\frac{5}{8} m\right)\left(-M_{\text {con }}\right)\right],
\end{array}
$$

To realize this dependence, turn to Figure $2, b$ and 3 . The efforts of $\mathrm{Tb}(\mathrm{z})$ and sr are not included in the design scheme, because their projections onto the $\mathrm{X}$ axis are zero, and in the moment equation their shoulders are negligible.

Displacements are determined by the methods of structural mechanics in sections I-I, II-II, IIIIII with the use of Figure 3 and are equaled to:

$$
\zeta_{b u}=\frac{1}{3} \sum_{i=1}^{5}\left(\frac{P_{i}^{2} \partial C_{i}}{\partial A}-C_{i} P_{i} \frac{\partial P_{i}}{\partial A}\right)
$$

where $\quad M_{4}=t+\frac{5}{8} m ; \quad M_{5}=h_{c r c} ; \quad M_{6}=\frac{2}{3} t+\frac{5}{8} m ; \quad M_{7}=h_{c r c}-\frac{1}{3} t ; \quad M_{8}=h_{c r c}-t-\frac{5}{8} m$.

The terms are excluded containing the areas of single diagrams and in sections III-I and III-II (in view of their smallness) in equations (16)(18).

The displacements associated with the rotation of the setting by the angle $\mathrm{f}_{2}$ are determined from simple geometric relations:

$$
\begin{gathered}
\delta_{\mathrm{I}}=\varphi_{2} h_{c r c} ; \\
\delta_{\text {II }}=\varphi_{2}\left(h_{c r c}-\frac{1}{3} t\right) ; \\
\delta_{\text {III }}=\varphi_{2}\left(h_{c r c}-t-\frac{5}{8} m\right) .
\end{gathered}
$$

Here the angle $f_{2}$ is determined from the angle of the neutral axis rotation of the reinforced concrete element from the equation:

$\varphi_{2}=\varphi_{1}+\Delta \varphi$,

where $\Delta \varphi$ - is the difference between the angles of rotation console between points $\mathrm{O}$ and $\mathrm{B}$ (Figure $2, a)$.

The angle $\varphi_{1}$ is determined by the curvature of the reinforced concrete element. In this case, it is necessary to subtract that part of the angle of rotation of the neutral axis, which is caused by the deformation of the reinforced concrete element before the crack's appearance. Assuming an additional premise that the crack appears immediately spreads over the length of hcrc with respect to the double-cantilever element, we can write:

$$
\varphi_{1}=\frac{t}{h_{0}}\left(\varepsilon_{s} \psi_{s}+\varepsilon\right)-\frac{t M_{\text {crc }}}{0,85 E_{b} I_{\text {red }}} .
$$

To determine the angular difference $\Delta \varphi$, refer to Figure 4:

$$
\Delta \varphi \approx-\frac{1}{E_{b} I_{\text {red }}} \frac{M_{0}+M_{c r c}}{2} m .
$$




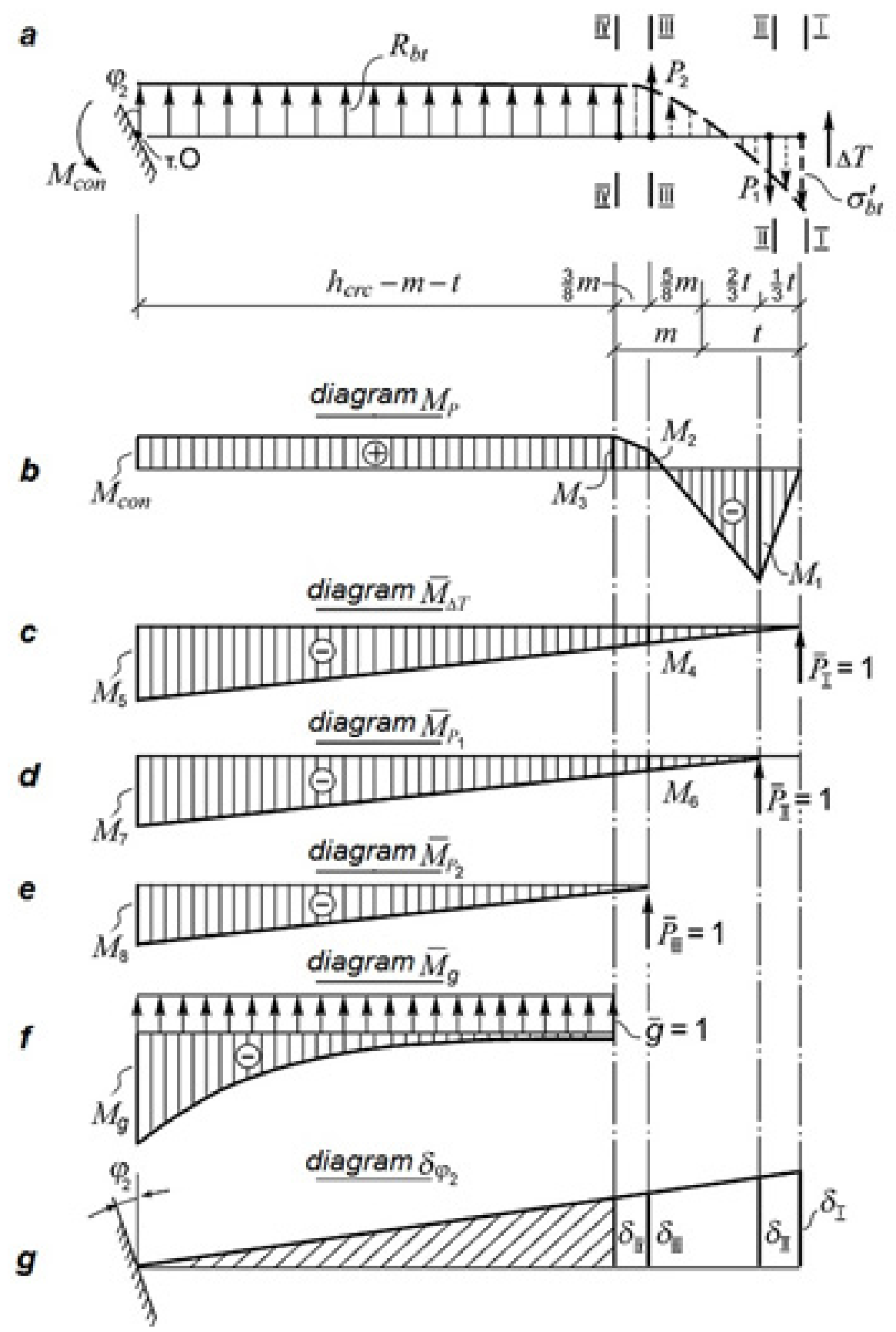

Figure 3: The calculated cantilever scheme (a), the load diagram of the moments (b), the unit diagrams of the moments (c)-(f) and the displacement diagram from the rotation of the seal by the angle $\varphi_{2}(g)$

Thus, all the parameters included in the formulas (16)-(18) and, consequently, the displacements I, II, III and the rotation angle $\varphi_{2}$ are determined. Passing to the corresponding compliances, we get:

$$
\begin{aligned}
C_{\text {I }} & =\frac{2 \Delta_{\mathrm{I}}}{\Delta T} ; \\
C_{\text {II }} & =\frac{2 \Delta_{\text {II }}}{-P_{1}} ; \\
C_{\text {III }} & =\frac{2 \Delta_{\text {III }}}{P_{2}} ;
\end{aligned}
$$

$$
C_{0}=\frac{2 \varphi_{2}}{M_{\text {con }}}
$$

where $P_{1}=0,5 \sigma_{b t}^{\prime} b t ; \quad P_{2}=\frac{2}{3} R_{b t} b m$

The compliance, corresponding to the distributed load, can be expressed as:

$$
C_{q}=\frac{2 A_{\Delta q}}{q},
$$


where $A \Delta q-$ the area of the displacement diagram in the distributed load place.

For determining $A \Delta q$, we return to Figure 3 :

$A_{\Delta q}=A_{\delta \varphi}+\frac{1}{E_{b} I_{\text {con }}} \frac{1}{3} M_{g}\left(h_{c r c}-t-m\right)\left(-M_{\text {con }}\right)$.

Here, $\quad M_{g}=\frac{1}{2}\left(h_{c r c}-t-m\right)^{2}$.

The parameter $\mathrm{A}$ is determined by the formula (Figure 4):

Here

$\delta_{I V}=\varphi_{2}\left(h_{c r c}-t-m\right)$.

After algebraic transformations, formula (15) reduces to the form:

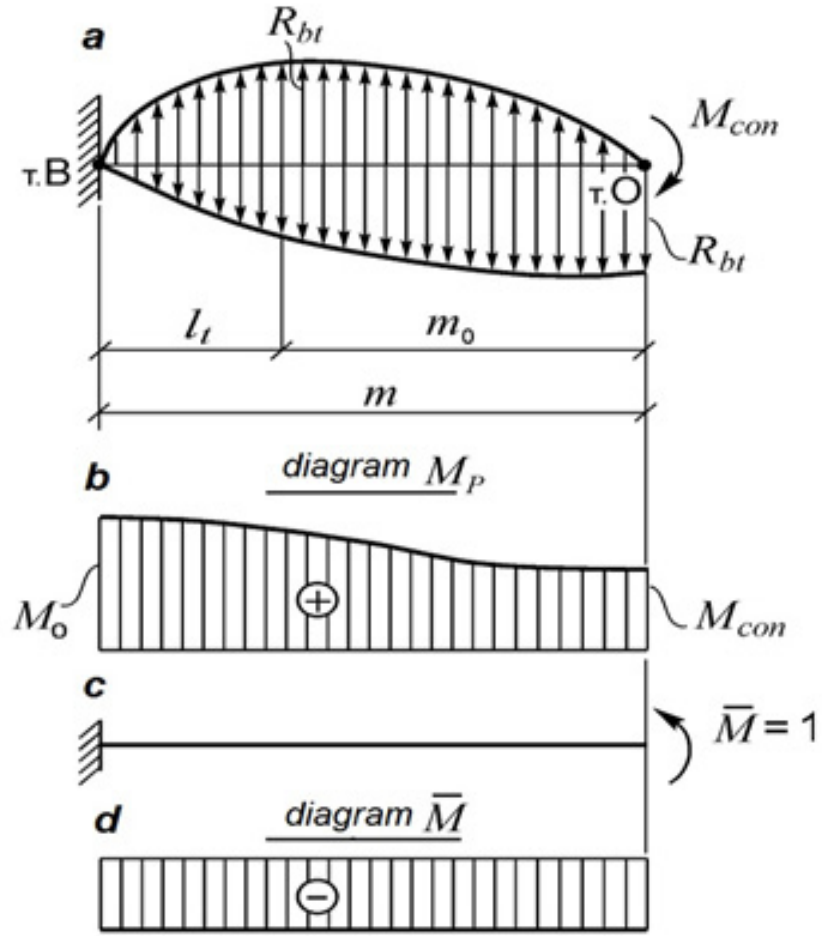

Figure 4: The calculation scheme for determining the difference between the angles of rotation between the points $O$ and $B(a)$, the load diagram of the moments (b), the loading by the unit moment (c), and the unit diagram of the moments $(d)$

\section{CONCLUSIONS}

Performing term-by-term differentiation, according the fact that the derivatives and tend to zero (in view of the small change in the parameters $m$ and $\mathrm{t}$ ), and making algebraic transformations [5], we obtain a nonlinear differential equation of the first degree that can be solved with respect to the derivative. Its solution is sought in the same way as for the equation in complete differentials. The integration constant is sought from the con- dition that, in the absence of a crack, that is, at hcrc $=0$, the tangential force $\Delta T=$ Greqelbt. Here eqel are the relative mutual displacements of reinforcement and concrete in section $t$ before the appearance of cracks; GT - module of mutual displacement of reinforcement and concrete.

As a result, after simplifying the general integral of the equation, we obtain:

$\Delta T=\frac{h_{c r c}\left(\eta_{15}-\eta_{5}-\eta_{8}\right)-G_{\tau} \varepsilon_{q, e l} b t \eta_{2}+0,5 h_{c r c}^{2} \eta_{14}}{\eta_{2}+\eta_{c r c}\left(\eta_{1}+2 \eta_{7}-\eta_{8}\right)}$ 
where $\mathrm{y} 1, \mathrm{y} 2, \mathrm{y} 5, \mathrm{y} 7, \mathrm{y} 8, \mathrm{y} 14, \mathrm{y} 15$ are expressed in terms of parameters $|1, K| 12,, y 1, y 2, A 1, A 0$ and are functions of Rbt, Eb, $\mathrm{m}, \mathrm{t}, \mathrm{b}$, Ican. In addition, the parameter $\mathrm{A} 0$ depends on the angle of rotation of the neutral axis y1 rod reinforced concrete element (Figure 4), and parameter y15 depends on the new concrete constant zbu.

This dependence makes it possible to find tangential stresses in the zone immediately adjacent to the crack. It is here, as shown by experimental and numerical studies, there is a sharp perturbation of tangential stresses, accompanied by their abrupt increase and sign change. In this case, the signs of normal stresses in concrete change (they become compressible from stretching), which is also confirmed in experiments [03, 05], although no explanation has been given to this phenomenon to date.

Here the reason is that, after the formation of cracks, the continuity of the concrete is disturbed and its deformation no longer obeys the laws of the continuous body. In zones adjacent to cracks, there is a concentration of deformations, which oversaturates the "need of the system" (consisting of concrete blocks and reinforcement for a given static scheme) in deformations. Thus, an additional deformation effect occurs in the cracks, which causes the effect observed in the experiments $[03,05,07]$. Given such a deformation effect in numerical experiments, we obtained a picture of a stress-strain state, similar to the experimental one. Consequently, the cause of the stress-strain state perturbation in the zones adjacent to the cracks is the additional deformation effect in the crack, which must be considered in the calculation [13, 14]. At the same time, the connection between the components of the stress-strain state in the perturbation zone with the specific energy of new crack surfaces formation, which is released in the predestruction zone.

As a result, it was obtained a new solution of the stress-strain state problem of the reinforced concrete structures in the zone immediately adjacent to the crack. The unknown parameters $\mathrm{m} 0$, Mcon, hcrc, t, It, $\Delta T$ are determined respectively from equations (2), (6), (7), (9), (3), (34).

\section{REFERENCES}

1) $\mathrm{ACl}$ Committee 446, Fracture Mechanics (1992) Fracture mechanics of concrete structures. Part I, State-of-Art Report (Edited by Z.P. Bažant), Elsevier Applied Science, London and New York: 1-140. [In English]

2) Zaytsev Yu. V. (1991). Fracture Mechanics for builders, High School Publ., Moskow, 288 p. [In Russian]

3) Golishev, A. B. and Kolchunov, V. I. (2009), The resistance of reinforced concrete, Osnova Publ., Kiev, 432 p. [In Russian]

4) Morozov E.N., Nikishkov G.P. (2010). Finite element method in fracture mechanics. Editorial URSS Publ., Moskow, 256 p. [In Russian]

5) Veruzhsky Yu. V., Kolchunov VI.I. (2005). Methods of reinforced concrete mechanics. NAU Publ., Kiev, 653 p. [In Russian]

6) Kolchunov VI.I., lakovenko I.A. (2009). The development of a two-cantilever element of the fracture mechanics for calculation the width of cracks opening in reinforced concrete structures. Bulletin of Civil Engineers. SPbGASU, St. Petersburg, № 4 (21), pp. 160-163. [In Russian]

7) Klueva N. V., Kolchunov VI.I., lakovenko I.A. (2014). The problem tasks of the fracture mechanics hypotheses development applied to the calculation of reinforced concrete structures. Kazan State University of Architecture and Engineering news, Vol. 3(29), pp. 41-45. [In Russian]

8) Kolchunov VI.I., lakovenko I.A. (2016). On the account of the disruption of continuity effect in reinforced concrete during the reconstruction of textile industry enterprises. Proceedings of high schools. Technology of textile industry. Vol. 3 (363), pp. 258-263. [In Russian]

9) Barenblatt G.I. (1993c). Some general aspects of fracture mechanics. In Modelling of Defects and Fracture Mechanics, Herrmann, G. (ed.), pp. 29-50. Springer-Verlag, Vienna, New York. [In English]

10) Sih G.C. (1973). Some basic problems in fracture mechanics and new concepts. Engineering Fracture Mechanics, Vol. 5, pp. 365-377. [In English]

11) Hillerborg A., Modeer M. and Petersson 
12) P.E. (1976). Analysis of crack formation and crack growth in concrete by means of fracture mechanics and finite elements, Cement and Concrete Research, 6, pp. 773-782. [In English]

13) Bažant, Z.P. and Oh, B.H. (1981). Concrete fracture via stress-strain relations, Report 81-10/665 c, Center for Concrete and Geomaterials, Northwestern University. [In English]

14) Xu S., Zhang X (2008). Determination of fracture parameters for crack propagation in concrete using an energy approach. Eng. Fract. Mech., Vol. 75: pp. 4292-4308.

15) Yehia N.A.B. (2009). Fracture mechanics approach for flexural strengthening of reinforced concrete beams. Engineering Structures, Vol. 31, Issue 2, pp. 404-416. [In English]

Paper sent to revision: 21.07.2017.

Paper ready for publication: 15.09.2017. 